DIMACS Serie日 in Discrete Mathematic:

and Theoretical Computer Science

\title{
In Vitro Selection for a OneMax DNA Evolutionary Computation
}

\author{
David Harlan Wood ${ }^{\dagger}$, Junghuei Chen ${ }^{\dagger}$, Eugene Antipov ${ }^{\ddagger}$, Bertrand \\ Lemieux, and Walter Cedeño
}

\begin{abstract}
Aspects of Evolutionary Computation, DNA computing, and in vitro evolution are combined in proposed laboratory procedures. Preliminary experimental results are shown. The traditional test problem for Evolutionary Computation known as the OneMax problem is addressed. The preliminary experimental results indicate successful laboratory "separation by fitness" of DNA encoded candidate solutions.
\end{abstract}

\section{Introduction}

Evolution is a concept of obtaining adaptation through the interplay of selection and diversity. Analogies from evolution have been used in both computing and molecular biology. These two areas are called respectively "evolutionary computation" and "in vitro evolution." From the beginning of DNA based computing to the present there have been calls $[\mathbf{1 1}, \mathbf{2 2}, \mathbf{2 7}]$ to carry out evolutionary computations using genetic materials in vitro. So far, there have been three such experiment designs proposed, including two in a recent DIMACS Workshop $[4,7]$. The very first design was presented about two years ago [10], but has not yet been carried out in the laboratory.

We design a laboratory implementation of an evolutionary computation. The crucial operation of physically separating DNA strands by their "fitness" is realized using 2- $\mathbf{d}$ denaturing gradient gel electrophoresis (2d DGGE). The interest is that candidate solutions are encoded in strands of DNA. Thus, trillions of candidate solutions have their fitness evaluated in parallel. Let us now comment on the possible variety of selection techniques usable after candidates have been physically separated by 2d DGGE. It is not clear if any of these directly correspond to any of the usual selection schemes from classical Evolution Computation. However, we are able to recover separated candidate solutions that are expected to be more fit, on average, than candidate solutions we discard. Our expectation is encouraged by preliminary experimental results included in this paper indicating that even a

1991 Mathematics Subject Classification. Primary 68Q40, 68U99, 68Q15.

†Partially supported by DARPA/NSF Grant No. 9725021.

${ }_{+}^{\ddagger}$ Partially supported an REU Supplement to DARPA/NSF Grant No. 9725021, and as a Howard Hughes Medical Institute Scholar. 
single round of selection significantly enhances the average fitness of a population of candidate solutions.

This paper is based in part on a paper [34] for the Genetic and Evolutionary Computation Conference. In the present paper we elaborate on the DNA laboratory aspects of our research. In an additional paper paper we plan to compare our techniques with those used for Evolutionary Computation on conventional computers. The laboratory designs and results discussed in this paper are still preliminary but incorporate (1) details of experimental design, (2) laboratory demonstration of selection and readout, (3) computations confirming alignment specificity, and (4) computer simulation of sample candidate DNA strands in $2 \mathbf{d}$ denaturing gradient gel electrophoresis.

\section{Evolutionary Computation Outline}

In this paper we identify aspects of evolutionary computations and in vitro evolution that we combine to address a OneMax problem, a traditional test case. Specifically, we choose Evolutionary Computation manipulating bitstrings using operations of pointwise mutation and crossover. These two operations can be performed by modifying and extending techniques from in vitro evolution.

"Evolutionary Computation" comes in many, many different varieties [3], but most are variations and/or elaborations on the following very loose outline. Evolutionary Computations typically cycle electronic computers through the following steps, maintaining a population of candidate solutions of a problem.

\section{Evolutionary Computation}

Begin with a diverse initial population, perhaps chosen randomly. Repeat the following steps until convergence.

1. Evaluate fitness of candidates.

2. Select the more fit candidates to breed and the less fit to be replaced.

3. Induce variation by breeding.

2.1. Evolutionary Computation Has Unclear Aspects. We are careful to make the following point. We do not take any stance on the virtues of any particular method of evolutionary computation. Instead, we aspire to provide the means for assessing some evolutionary computations using population sizes larger than is practical with conventional computers.

Evolutionary computation makes few assumptions and is ostensibly applicable to very broad classes of problems. Naturally, this makes it difficult to establish many provable guidelines. Just to list a few aspects which are sometimes unclear,

- How does one model fitness?

- How disruptive is crossover?

- What are the pros and cons of transposition, inversions, and introns?

- How long does it take evolutionary computations to converge?

- How does one recognize convergence?

\section{Implementing Evolutionary Computation Using DNA}

Several means of DNA computation have been addressed. The first was, of course, by Adleman [1, 2]. Recent overviews can be found in [15] and [21]. See also the DNA Computing bibliography of J. H. M. Dassen [9]. 
We anticipate computing time using DNA to be proportional to the number of generations required, but independent of population size. This motivates incorporating both pointwise mutation and crossover, and for that matter any evolutionary analogies that might reduce the number of required generations.

Modifications and extensions of current technology seem to suffice to implement crossover and pointwise mutation. However, selecting DNA strands for "breeding" is moderately challenging because one must physically separate DNA strands according to their "fitness."

3.1. DNA Attributes Suit Evolutionary Computation. Of all evolution inspired approaches, certain versions of Evolutionary Computation seem particularly suited to using DNA strands. This is because DNA strands can encode populations of bitstrings suitable for both crossover and pointwise mutation.

DNA computing techniques are desirable for Evolutionary Computation.

- The first main advantage is these techniques might process, in parallel, populations billions of times larger than is usual for conventional computers. this would be beneficial if larger populations might generate high-fitness individuals in fewer generations.

- The second main advantage is the massive information storage available using DNA. For example, a gram of DNA primers contains about $10^{21}$ bases. The information content is approximately $2 \times 10^{21}$ bits, greatly exceeding the 200 petabyte storage of all digital magnetic tape produced in 1995 [33].

- A third advantage is that methods for implementing crossover using DNA are possible as variations on "Sexual PCR" pioneered by Stemmer [28].

- An additional issue is that biolaboratory operations on DNA inherently involve errors. To some extent, errors may be regarded as noisy fitness evaluation. Such errors are sometimes tolerable when executing Evolutionary Computation [14], unlike when executing deterministic algorithms.

- Oversimplified estimates indicate [34] DNA computing techniques might compare favorably to supercomputers in some cases. These favorable cases include executing Evolutionary Computation having very simple fitness evaluations and very large populations of candidate solutions.

3.2. DNA Evolutionary Computation Compared to In Vitro Evolution. Evolutionary Computation are reminiscent of methods in molecular biology referred to as "in vitro evolution." Naturally, these methods use fitness criteria constrained to properties of biomolecular interest. Indeed, the necessity of finding methods to physically separate biological materials by "fitness" has determined which problems are addressed by in vitro evolution. In vitro methods suit some very important but relatively small classes of problems seeking ribozymes $[\mathbf{3 1}, \mathbf{2 3}, \mathbf{1 6}, \mathbf{2 9}]$, binding sites $[\mathbf{3 0}, \mathbf{2 9}]$, enhanced enzymes [26], etc.

In contrast to in vitro evolution, Evolutionary Computation uses unconstrained bitstrings as fitness criteria. Consider computations using bitstrings of length 100 . Such computations can, in principal, evolve a population of fixed size in such a way as to create any one of $2^{100} \approx 10^{30}$ possible outcomes. Thus, Evolutionary Computation methods using DNA can address larger, less specialized, classes of problems than does in vitro evolution.

Evolutionary Computation using DNA would be similar to conventional computers in that (virtually) all $10^{30}$ possible inputs and outputs would be equally 
suitable. In contrast, in vitro evolution is tailored to the very rare DNA sequences encoding biological functionality. For example, bitstrings might be realized as DNA strands having $100 \mathrm{As}$ and Gs. But as far as we know very few, if any, such DNA bitstrings code for biologically active functions. Or to put it another way, virtually all sequences are equally "meaningful" for the purposes of Evolutionary Computation. In contrast, in vitro evolution focuses on variations of the very rare DNA

sequences of biological or biochemical interest.

\section{The OneMax Problem}

Here we present a problem that is relatively simple to address using DNA implementations of Evolutionary Computation. The essence of our implementation is that more fit candidates strands of DNA can be physically separated from less fit candidates according to how well they match (hybridize with) "target" DNA strands.

The OneMax problem is a traditional test problem for Evolutionary Computation. It involves binary bitstrings of fixed length. An initial population (usually randomly generated) is given. The objective is to evolve some bitstrings to match a prespecified "target" (generally taken to be all 1s). The study of OneMax problems has lead to a number of insights. For example, see $[\mathbf{1 3}]$ and the references cited there.

4.1. Outline of DNA Implementation. We now outline a DNA implementation of the OneMax problem. The corresponding information is shown in Figure 1 . In the remainder of this section, details are given. Throughout this section, information is grouped in the following categories: (1) candidate pool, (2) fitness evaluation, (3) selection and (4) breeding.

Preliminary laboratory results are given later, in Section 5.

The implementation is given by the following outline. The same information, with a few added details, is shown in Figure 1.

DNA Evolutionary Computation for OneMax Problem

Begin with a diverse initial population of candidates.

Repeat the following steps until convergence.

1. Evaluate fitness by hybridizing to target strands and physically separate on a 2 -d gel.

2. Select and purify relatively more fit candidates to breed.

3. Amplify fit candidates with pointwise mutation and reserve a portion.

4. Breed candidates, using crossover.

5. Combine reserved and bred candidates, obtaining a new generation.

4.2. Design of Candidate Solutions and Target DNA Strands. Figure 2 shows our design. A target strand and a perfect candidate strand are shown in the figure. Imperfect candidate strands have a mixture of $40 \mathrm{Ts}$ and Cs instead of 40 consecutive Ts. At the $5^{\prime}$ end of all candidate strands there is a universal section known as the CLAMP section of the target strands. All $3^{\prime}$ ends of the candidate strands are extended by a universal TAIL sequence. The candidate strands are longer to facilitate eventual separation of target and candidate strands using denaturing gel electrophoresis. 
Initialize with Random candidates

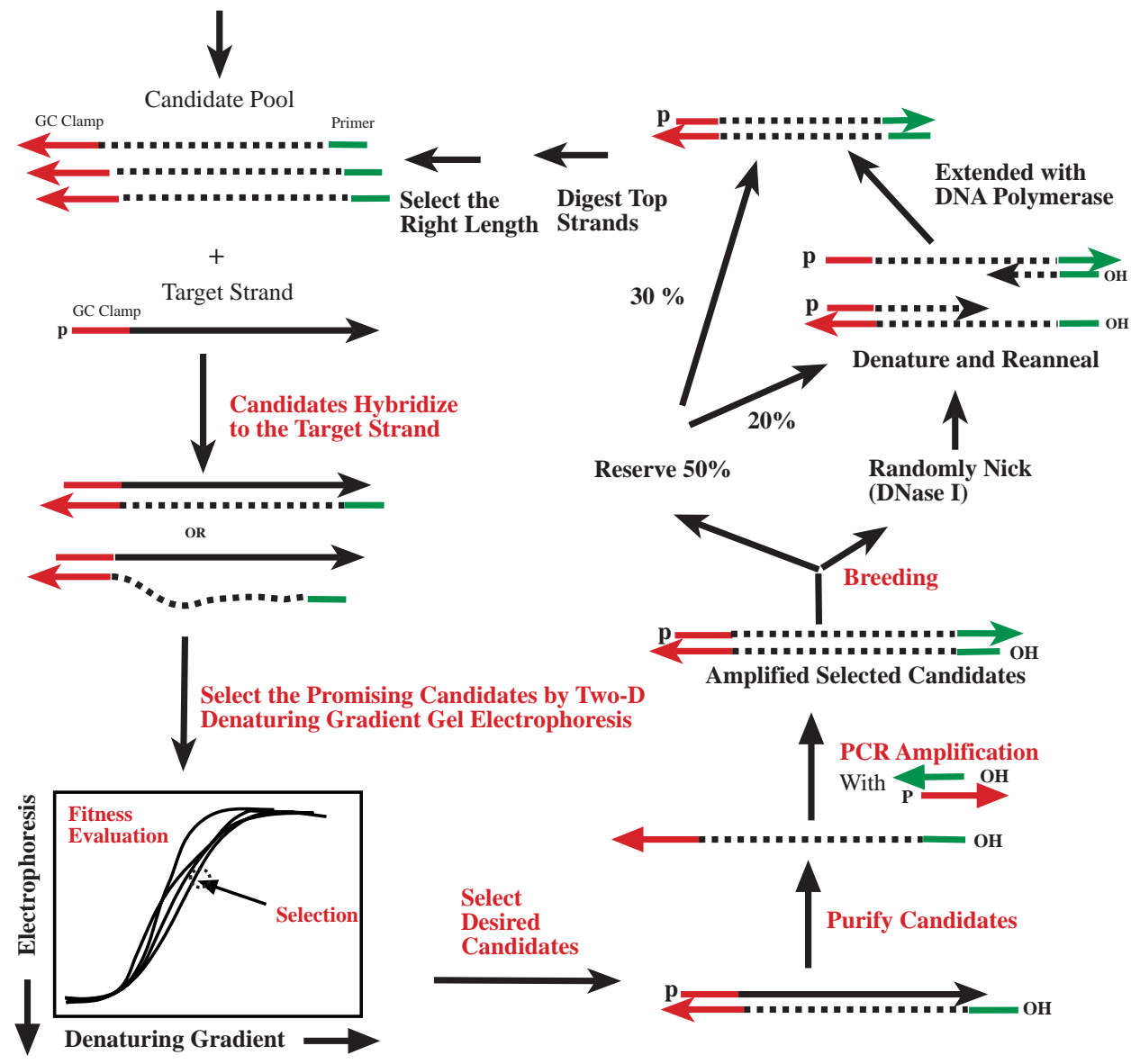

Figure 1. Outline of DNA implementation of Evolutionary Computation for OneMax problem. The candidate pool appears in the upper left. Selection using 2-d DGGE appears at the lower left. Purification and amplification of the relatively more fit candidate strands appears at the lower right. Breeding using crossover appears at the upper right.

Since candidate strands have known primer sites at both ends, they can be amplified by PCR. One primer we will call the TAIL-primer, which is the reverse complement of the TAIL. The other primer is simply the CLAMP sequence.

The CLAMP and TAIL sequences have been designed to encourage correct alignment of the PCR primers and to avoid sticking where they are not supposed to stick. Figure 3 concerns how misaligned primer may stick (hybridize) to candidate strands. Figure 3 shows melting temperatures $\mathrm{T}_{\mathrm{m}}$ (a measure of the strength of hybridization) at all possible alignments of the CLAMP and the TAIL-primer. These short strands are tested against a full-length candidate strand. This figure and the next was generated using the BIND software [17] from MIT, for which we have a license for limited use. 


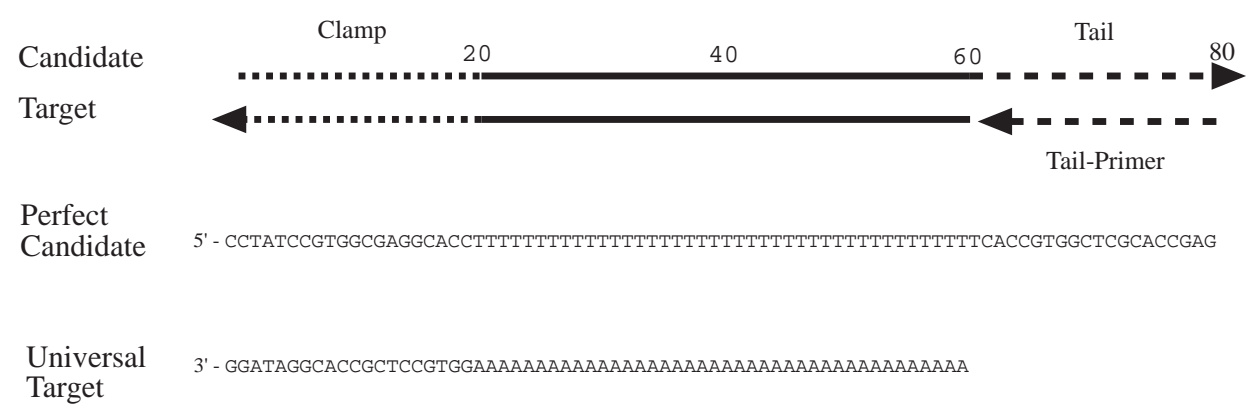

Tail Primer

Figure 2. Design of target and candidate strands. Perfect candidates have all Ts from position 21 to position 60 . Imperfect candidates would have a mixture of Ts and Cs in place of the 40 Ts in the perfect candidate.

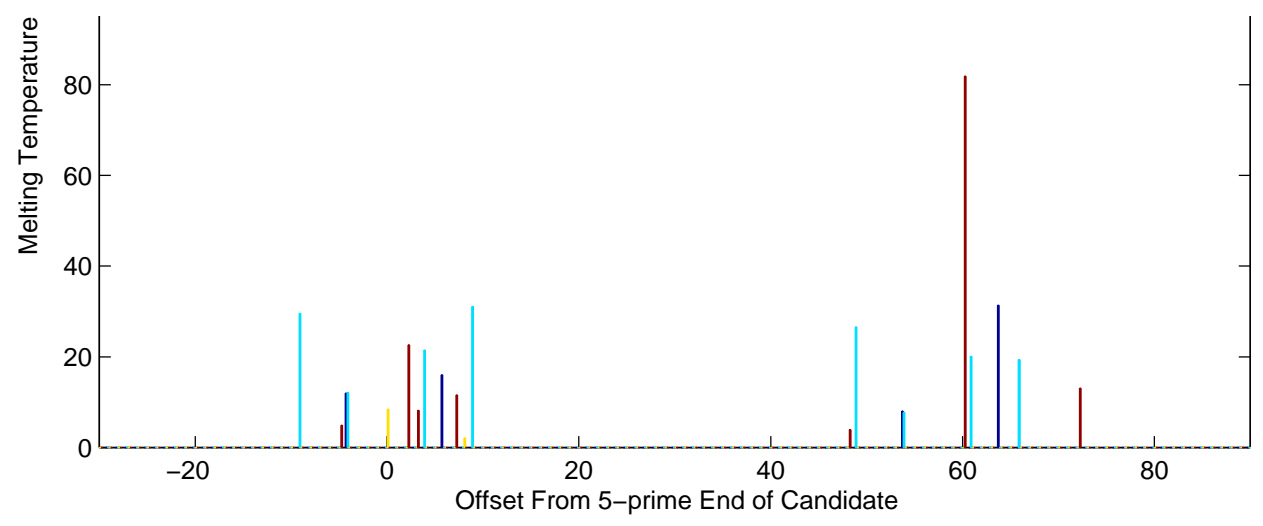

Figure 3. At an offset of 60 , the correctly aligned TAIL-primer sticks tightly to the candidate strand, providing a melting temperature of 82 degrees. Misalignments of the CLAMP and the TAILprimer, can only stick weakly with melting temperatures of 31 degrees or less.

Figure 4 concerns how misaligned primer may stick (hybridize) to complements of candidate strands. Figure 3 shows melting temperatures $\mathrm{T}_{\mathrm{m}}$ at all possible alignments of the CLAMP and the TAIL-primer. These short strands are tested against the full-length reverse complement of a candidate strand.

4.3. Fitness Evaluation by DGGE Physical Separation of DNA. Our fitness evaluation is carried out in the laboratory using so-called 2-d denaturing gradient gel electrophoresis (DGGE) [18]. Let us first review the nature of DGGE. Figure 5 shows DGGE from our laboratory having perfect candidates combined with target strands. The target strands hybridize (stick) to the perfect candidate strands. Each candidate is extended by a universal TAIL at its $3^{\prime}$ end. The mixture of hybridized strands is placed uniformly along the top of the gel. The hybridized 


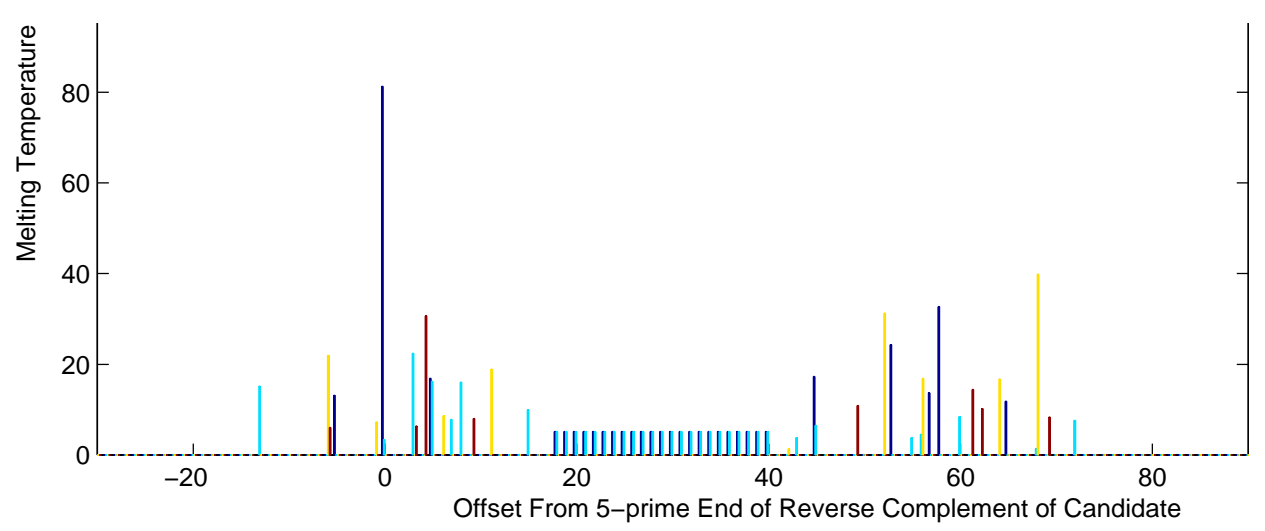

Figure 4. At an offset of 0 , the correctly aligned CLAMP sticks tightly to the reverse complement candidate strand, having a melting temperature of 81 degrees. Misalignments of the CLAMP and the TAIL-primer, can only stick weakly with melting temperatures of less than 40 degrees.

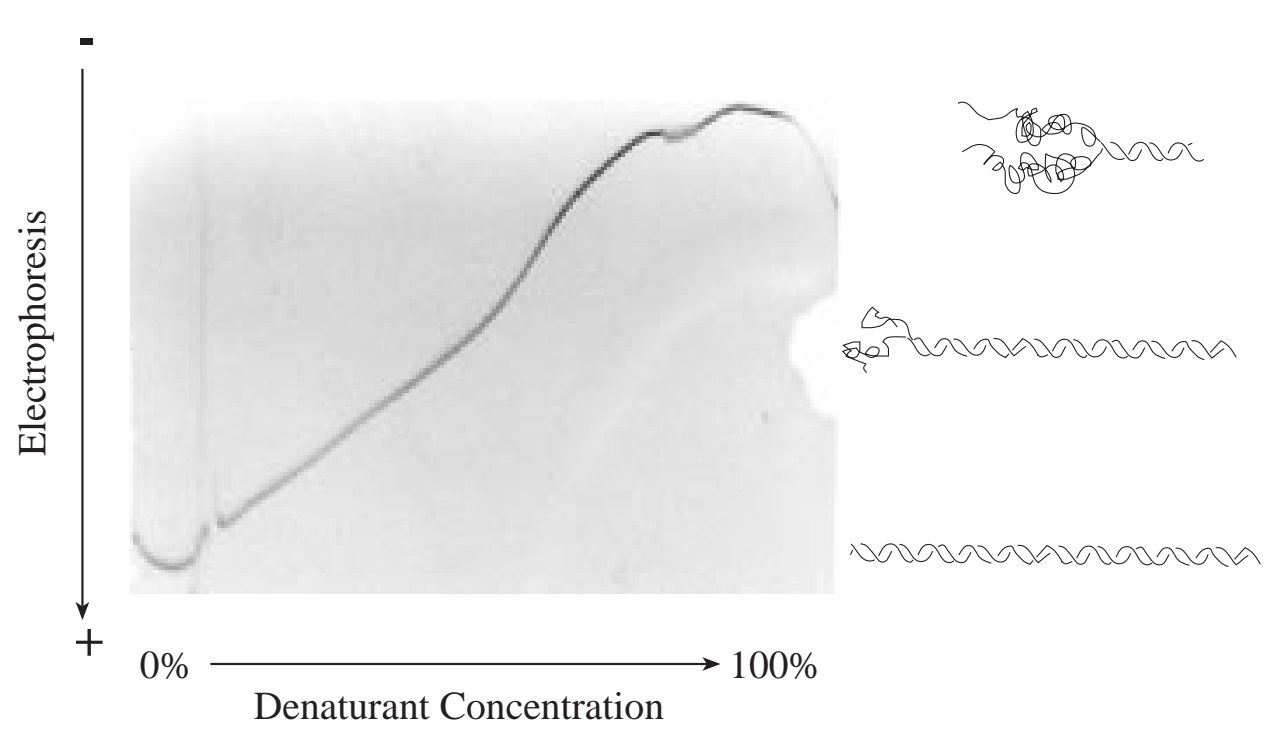

Figure 5. DGGE using perfect candidates. DNA strands move downward from a reservoir at the top of the figure. The speed of vertical strand migration is retarded as strands come apart (denature) as shown schematically on the right edge of the figure.

strands travel vertically downward in the gel as a result of an applied electric field. However, their speed of migration is determined by their initial placement from left to right; that is, by how strongly they are denatured (pulled apart). On the left, where no denaturant is encountered, the strands move relatively quickly downward. In the center, they move more slowly because they encounter intermediate denaturing. At the extreme right, the stands are able to move only very slowly because 
the strands are almost completely pulled apart except in the more resistant CLAMP region.

An important fact for DNA computing is that DGGE can detect a single base mismatch. Indeed, this was an early application of DGGE in molecular biology $[18]$.

4.4. Selection of Relatively More Fit Candidate Solutions. Selection is done by literally cutting out a portion of the 2- $\mathbf{d}$ gel and extracting the DNA strands from it. The most fit candidates are presumably lowest on any vertical line.

This allows a wide latitude for selection criteria. Experience in Evolutionary Computation computing demonstrates the desirability of maintaining genetic diversity to prevent the loss of genetic materials which may be needed in later stages of evolution.

4.5. Amplification With Mutation via PCR. The purified candidate strands are amplified by PCR, which also induces pointwise mutation at at a rate of about $10^{-4}$ nucleotide per cycle when Taq DNA polymerase is used [12]. Special PCR protocols can be used to induce pointwise mutation at intermediate rates $10^{-2}$ to $10^{-4}[5,6,8,19,20,32,35]$.

Two primers are used. One primer we will call the TAIL-primer, which is the reverse complement of the TAIL. The other primer is a copy of the CLAMP. These two primers are chosen to produce repeated doubling of copies of the candidate strands (and their complements). In contrast, target strands will not double in number because they can hybridize with only one of the two primers, namely the CLAMP primer. Extension of this primer can form a complementary copy of the target. We see that candidate strands and their complements amplify at an exponential rate, doubling in number with each PCR cycle. They would increase a million-fold in 20 PCR cycles. But any target strands present can only make complementary target strands, and only at a linear rate. They would increase twenty-fold in 20 PCR cycles. It is important to note that these complements of target strands are both rare and short compared to candidate strands.

4.6. Purification of Mutated Candidates. The PCR product can be purified by length using conventional denaturing gel electrophoresis. This gets rid of any target strands and complements of target strands because these will be shorter than the candidates and their complements. Any excess primers are also eliminated. The purified candidates and their complements and be further amplified with mutation using additional primers and PCR. A relatively large quantity is generated to allow for the very low yield expected from the subsequent crossover step.

A portion of the double stranded product is temporarily reserved; the remainder is used for crossover.

4.7. Breeding Using Single Point Crossover. The double stranded product to be used for breeding is partially digested with DNase $I$ to mostly nick (cut only one strand) at random locations about once per strand. The nicked strands are combined with a similar amount of reserved unnicked strands. The mixture is denatured (strands are melted apart) and allowed to reanneal forming new combinations. Many, many possible configurations] could be formed. But among these, some will have portions of complements of candidate strands annealed at many positions to a $5^{\prime}$ end of a candidate strand. The TAIL of the candidate strands is 
designed to enforce alignment of such hybridizations. A typical desirable hybridization is illustrated in the upper right corner of Figure 1. By adding DNA Polymerase $I$, the partial candidate strand is extended to a full length candidate. In this process it combines its genetic information with that encoded in the intact strand. DNA Polymerase $I$ is used because it will remove any annealed fragments in its way as it proceeds in the $5^{\prime}$ to $3^{\prime}$ direction. This ensures the maximum possible extension.

The net result is single point crossover. The offspring candidate strand produced by the polymerase has a block of genetic information from one parent followed by another block from a different parent. In particular, our single point crossover extends results due to Stemmer $[\mathbf{2 8 ,} \mathbf{2 5}]$.

Single point crossover is a common approach used in Evolutionary Computation, but multiple point crossover can be achieved by iterating the above procedure.

4.8. Purification to Select Candidate Strands. The reaction products from crossover are combined with the reserved material. Purification by length (using denaturing gel electrophoresis) completes the breeding operation.

4.9. The New Generation. The new generation is now complete and ready to be processed.

4.10. Readout of Candidate Pool. We want to monitor the generation-togeneration progress of our in vitro evolution. We assume we are given a pool of candidate strands. (Any contaminating complementary strands, or contaminating target strands, will not affect the readout results.)

4.10.1. Estimation of Average Fitness. Figure 6 shows the method of generating radiolabeled primer extensions for readout. Our method is a variation on the Sanger method of DNA sequencing.

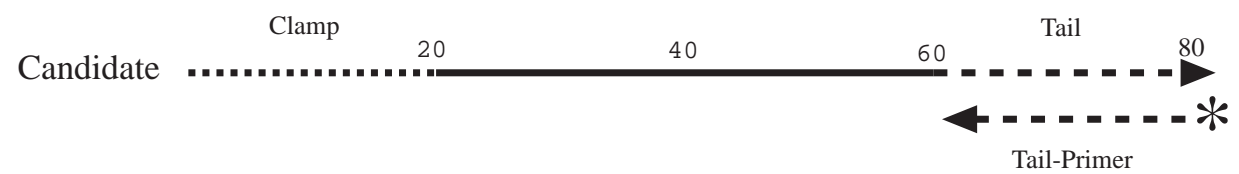

FigURE 6 . Readout of a candidate strand by means of primer extension. A radiolabeled TAIL-primer is annealed to the candidate strand and extended by Taq polymerase. With a certain probability, primer extension may halt when a $\mathrm{C}$ is encountered.

A large number of primers complementary to the TAIL section of candidates are radiolabeled and added to the pool of candidates. By first raising then lowering the temperature, each candidate preferentially anneals to a primer. The primer is extended in the $3^{\prime}$ direction by including Taq polymerase. However, we use only the nucleotides dATP together with a mixture of dGTP and ddGTP. On one hand, primer extension does not stop upon encountering a $\mathrm{T}$ in a candidate. This is because the nucleotide A, complementary to T, is contributed by dATP and incorporated at that point. On the other hand, primer extension can stop upon encountering a $\mathrm{C}$ in a candidate. This is because there is a mixture of dGTP and ddGTP. Since G is complementary to C, either dGTP or ddGTP can contribute a complement to $\mathrm{C}$. But whenever ddGTP is used, the strand can not then be extended any further. 
The result is: for each candidate strand, we obtain a primer extended by some number of bases. Each temperature cycle extends a radiolabeled primer on each candidate strand. The resulting extended primers can be separated by their various lengths and read out by using denaturing gel electrophoresis. This indicates the average density and location of the $\mathrm{Cs}$ in the candidate strands. Thus, a estimate of the average fitness of a candidate pool can be obtained.

\section{Experiment Demonstrating Selection and Readout}

5.1. Candidate Solutions Used for Readout. In our experiments the 40 variable positions between the CLAMP and the TAIL of each imperfect candidates are independently chosen to be Ts with probability .8 or Cs with probability .2 . Figure 7 shows how the average total number of Ts in each candidate varies in the randomly generated population of candidates.

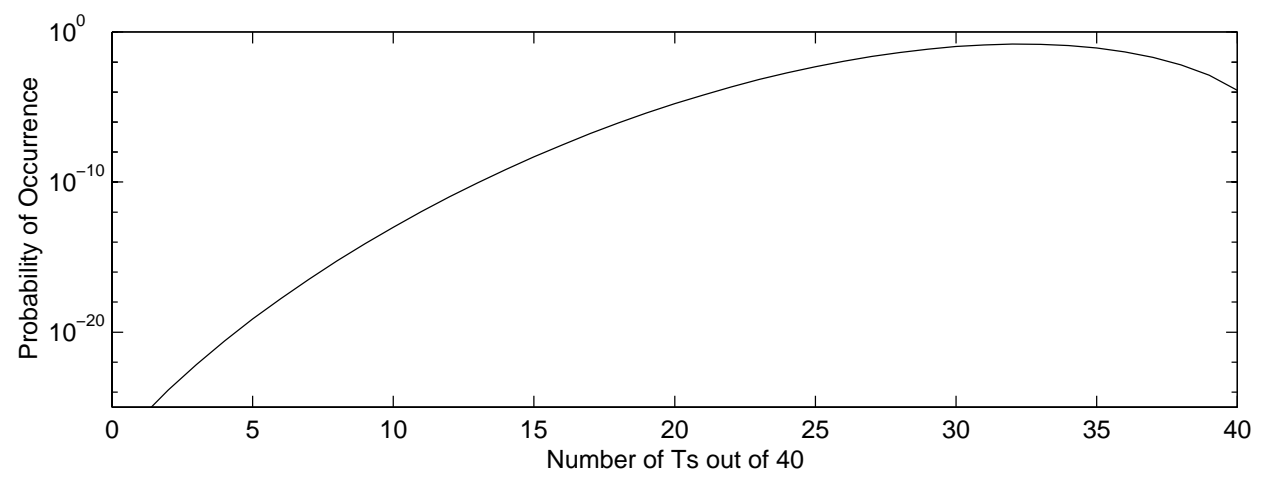

Figure 7 . Relative frequency as a function of the number of Ts. The case shown is a randomly generated population of candidates having 40 bases which consist of $80 \%$ Ts and $20 \% \mathrm{Cs}$.

5.2. Experimental Fitness Evaluation by DGGE Physical Separation of DNA. Recalling the nature of 2d DGGE discussed in section 4.3, we heuristically reason about a mixture of targets and imperfect candidates. We expect that everywhere across the gel the candidate strands that best match (hybridize to) the targets will migrate downward relatively faster. In fact, imperfect matches exhibit vertical spreading in our experiments. See Figure 8 . We assume that on any vertical line the most fit candidates are lowest on the 2d DGGE gel. However, the nature of variation from left to right is not clear. In our preliminary experiments, we have selected from several places at the lower edge of the 2d DGGE in Figure 8 and combined the extracted DNA. Further experiments will be needed to optimize a selection strategy.

Using the on-line POLAND software, we obtain estimates of the mobility of our particular candidate design, having (selected) mismatches. Figure 8 shows DGGE predictions from the PoLAND software [24].

5.3. Experimental Readout of the Result of First Round of Selection. Figure 9 contains data from a preliminary experiment demonstrating readout before and after selection. Deliberate overexposure of the lower half of the gel allows detail 

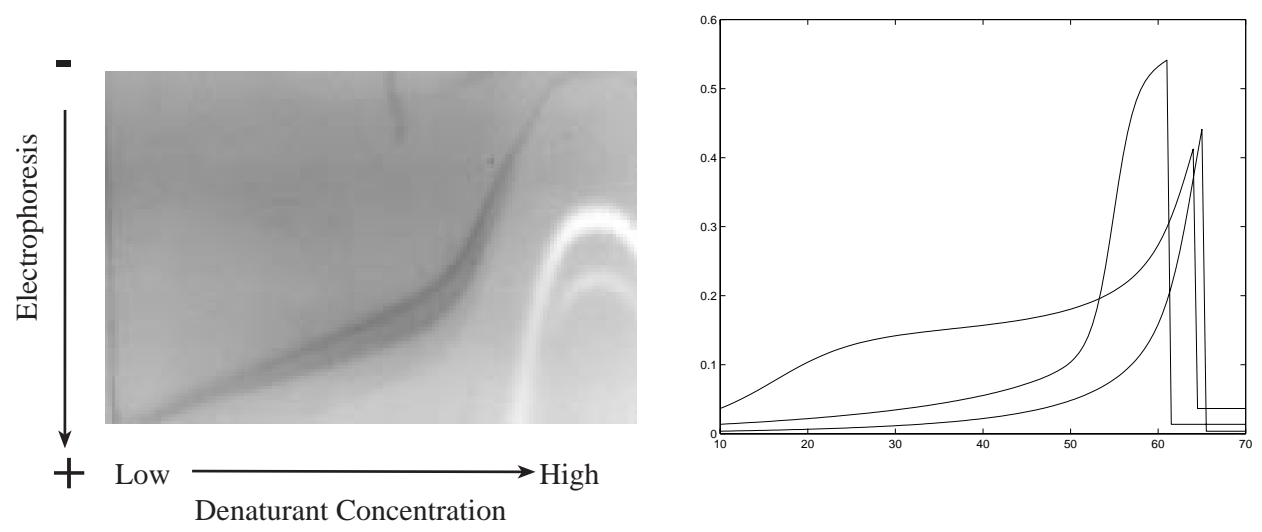

Denaturant Concentration

Figure 8. DGGE vertically separates imperfect candidates. The speed of vertical strand migration is retarded as strands come apart (denature). This is due to two factors: increasing denaturant concentration and decreasing quality of target-candidate matching (hybridization). On the right, DGGE predictions for selected imperfect candidates using the PoLAND software. At $50^{\circ}$, the curves correspond, from the bottom up, to perfect candidate, single mismatch at position 31 , and single mismatch at position 50 .

in the upper half to be observed. Lane 2 of the gel shows primer extensions for the initial population of randomized candidate strands. Lane 3 shows primer extensions for the population resulting from the first selection.

When a $\mathrm{C}$ is encountered during primer extension, there is a probability, call it $p$, of termination. That is to to say,

Probability of terminating $=p$ Probability of encountering a C.

Thus when the probability of encountering a $\mathrm{C}$ is a uniform constant,

Probability of terminal length $k=p[1-p \text { Probability of encountering a } \mathrm{C}]^{k-1}$.

Recall that for the initial population every position between 20 and 60 has .8 probability of being a $T$ and .2 probability of being a $C$. Using this fact together with the previous equation, we have

$$
\text { Probability of terminal length } k=p[1-0.2 p]^{k-1} \text {. }
$$

In summary, long extensions for the initial population are exponentially decreasing in likelihood. This corresponds exactly to the question "What is the frequency of observing runs of all tails using a fixed but biased coin?"

For the initial population the graphical readout in Figure 9, aside from the overexposed left half, shows the expected behavior of exponentially decreasing numbers of long extensions.

After the first selection, the right half of the graphical readout in Figure 9 shows that Cs are now much less common, allowing longer extensions. That is to say, no longer is the probability of encountering a $\mathrm{C}$ given by a uniform 0.2. Clearly, Cs are now much less likely, and they are no longer uniformly distributed.

The pool of candidates has been dramatically altered by the first round of selection. Now one observes many more long primer extensions. Therefore, the strands 
34

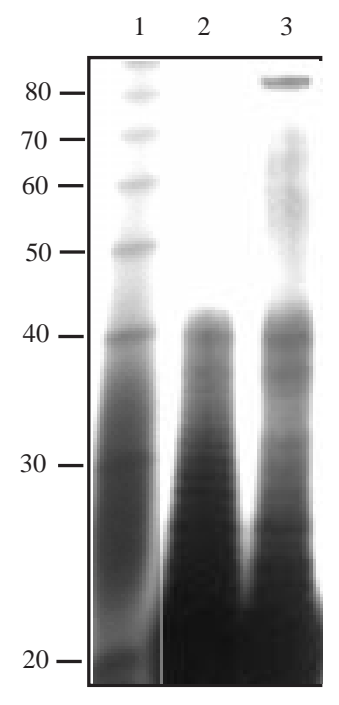

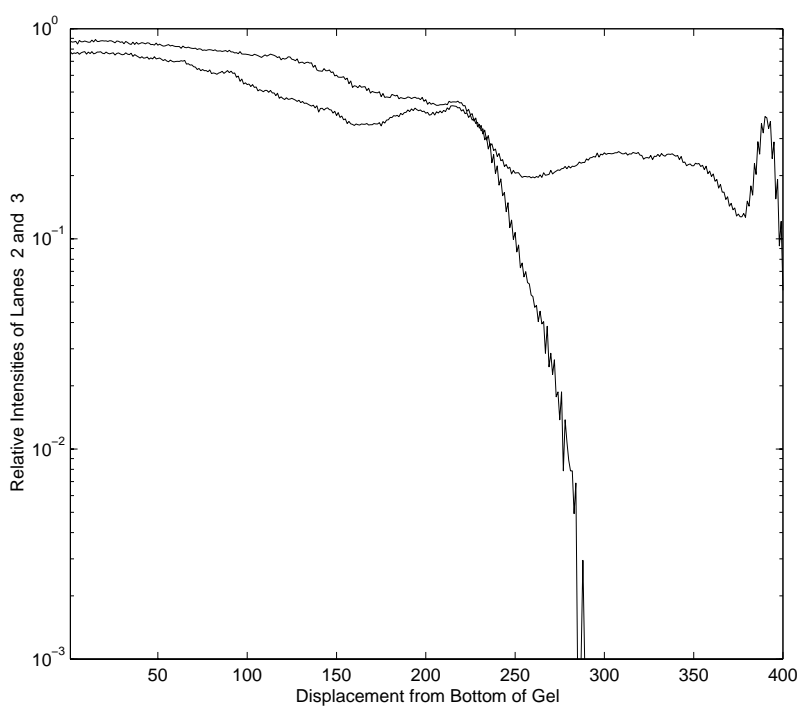

Figure 9. The gel on the left: Lane 1 contains a reference set of strands differing in length by 10 bases. Lane 2 is a readout generated by the pool of initially random candidates. Lane 3 is a readout generated after the first round of selection. The graph on the right: A logarithmic scan of Lanes 2 and 3. The uniform distribution of $\mathrm{Ts}$ in the initial population leads to exponential decay with increased length. After the first selection, Ts are much more rare which allows full length extensions.

being read out represent much more nearly homogeneous sequences. This represents significant progress toward our goal of evolving some completely homogeneous strands, which would represent solutions to the OneMax problem. Relatively many primer extensions run to the maximum possible length in Figure 9. That is to say, it appears that solutions of the OneMax problem have been selected. Regrettably, not all the full length extensions need represent perfect solutions because of unwanted substitutions by the polymerase. However, these substitutions can be made relatively rare. In any case, though, substitution effects are equally present in Lanes 2 and 3. It is the comparison of these two lanes that demonstrates the significant improvement resulting from the first round of selection. However, it must be said that the experiment shown in Figure 9 is still only preliminary and is in need of further refinement and calibration.

\section{Discussion}

Although much work remains to be done, it seems clear that even a single round of selection using 2d DGGE is capable of significantly enhancing the "fitness" within a population of candidate solutions of the OneMax problem. Since candidate solutions are encoded in strands of DNA, we are dealing with populations billions of times larger than is the usual practice with conventional computers. We reemphasize that candidate solutions in the entire population have their fitness evaluated at the same time. Separation by fitness is merely a single laboratory step. 
Of course, laboratory steps are slow compared to computer cycle times, but DNA can represent populations so large that their computational power can compare favorably with supercomputers [34].

\section{References}

[1] Leonard M. Adleman. Molecular computation of solutions to combinatorial problems. Science, 266:1021-1024, November 1994.

[2] Leonard M. Adleman. Computing with DNA. Scientific American, 279(2):54-61, August 1998.

[3] Thomas Bäck, David B. Fogel, and Zbigniew Michalewicz, editors. Handbook of Evolutionary Computation. Institute of Physics Publishing, Philadelphia, 1997.

[4] Thomas Bäck, Joost N. Kok, and Grzegorz Rozenberg. Evolutionary computation as a paradigm for DNA-based computing. In Laura Landweber, Erik Winfree, Richard Lipton, and Stephan Freeland, editors, Preliminary Proceedings DIMACS Workshop on Evolution as Computation, pages 67-88, DIMACS, Piscataway NJ, January 1999. Available on request from DIMACS. Paper found at URL: http://www.wi.LeidenUniv.nl/ joost.

[5] R. Craig Cadwell and Gerald F. Joyce. Randomization of genes by PCR mutagenesis. PCR Methods and Applications, 2:28-33, 1992.

[6] R. Craig Cadwell and Gerald F. Joyce. Mutagenic PCR. In Carl W. Dieffenbach and Gabriela S. Dveksler, editors, RCP Primer: A Laboratory Manual. Cold Spring Harbor Laboratory Press, Plainview, New York, 1995.

[7] Junghuei Chen, Eugene Antipov, Bertrand Lemieux, Walter Cedeño, and David Harlan Wood. DNA computing implementing genetic algorithms. In Laura Landweber, Erik Winfree, Richard Lipton, and Stephan Freeland, editors, Preliminary Proceedings DIMACS Workshop on Evolution as Computation, pages 39-49, DIMACS, Piscataway NJ, January 1999. Available on request from DIMACS. Paper found at URL: http://www.cis.udel.edu/ wood/papers/DIMACS_99.ps.

[8] R. Cheynier, S. Gratton, J. P. Vartanian, A. Meyerhans, and S. WainHobson. G $\rightarrow$ A hypermutation does not result from polymerase chain reaction. AIDS Research and Human Retroviruses, 13(12):985-986, August 10, 1997.

[9] J. H. M. Dassen. A bibliography of molecular computation and splicing systems. HTML source: http://www.wi.LeidenUniv.nl/ jdassen/dna.html, BibTeX source: http://www.wi.LeidenUniv.nl/ jdassen/dna.bib. This bibliography is also hooked into http://liinwww.ira.uka.de/bibliography/, The Collection of Computer Science Bibliographies.

[10] R. Deaton, R. C. Murphy, J. A. Rose, Max H. Garzon, Donald R. Franceschetti, and S. E. Stevens Jr. A DNA based implementation of an evolutionary search for good encodings for DNA computation. In IEEE International Conference on Evolutionary Computation, pages 267-271, Indianapolis, Illinois, April 13-16, 1997.

[11] Alan Dove. From bits to bases: Computing with DNA. Nature Biotechnology, 16(9):830-832, September 1998.

[12] K. A. Eckert and T. A. Kunkel. High fidelity DNA synthesis by the Thermus aquaticus DNA polymerase chain reaction. Nucleic Acids Research, 18:3739-3744, 1990.

[13] Philippe Gigure and David E. Goldberg. Population sizing for optimum sampling with genetic algorithms: A case study of the Onemax problem. In John R. Koza, Wolfgang Banzhaf, Kumar Chellapilla, Kalyanmoy Deb, Marco Dorigo, David B. Fogel, Max H. Garzon, David E. Goldberg, Hitoshi Iba, and Rick Riolo, editors, Genetic Programming 1998: Proceedings of the Third Annual Conference, pages 496-503, University of Wisconsin, Madison, Wisconsin, USA, 22-25 July 1998. Morgan Kaufmann.

[14] David E. Goldberg, Kalyanmoy Deb, and James H. Clark. Genetic algorithms, noise, and the sizing of populations. Complex Systems, 6(4):333-362, August 1992.

[15] Tino Gramß, Stephan Bornholdt, Michael Gramß, Melanie Mitchell, and Thomas Pellizzari. Non-Standard Computation. Wiley-VCH, Weinheim, 1998

[16] Rachel Green, Andrew D. Ellington, David P. Bartel, and Jack W. Szostak. In vitro genetic analysis: Selection and amplification of rare functional nucleic acids. Methods, 2:75-86, 1991.

[17] Alexander J. Hartemink and David K. Gifford. Thermodynamic simulation of deoxyoligonucleotide hybridization for DNA computation. In Harvey Rubin and David Harlan Wood, editors, DNA Based Computers III: DIMACS Workshop, June 23-25, 1997, volume 48 of 
DIMACS series in discrete mathematics and theoretical computer science, pages 25-37, Providence, 1999. American Mathematical Society.

[18] L. S. Lerman, K. Silverstein, and E. Grinfeld. Searching for gene defects by denaturing gradient gel electrophoresis. Trends in Biochemical Sciences, 172(3):89-93, 1992.

[19] M. M. Ling and B. H. Robinson. Approaches to DNA mutagenesis: An overview. Analytical Biochemistry, 254(2):157-178, December 15, 1997.

[20] J. L. LinGoerke, D. J. Robbins, and J. D. Burczak. PCR-based random mutagenesis using manganese and reduced dNTP concentration. Biotechniques, 23(3):407-, September 1997.

[21] Carlo C. Maley. DNA computation: Theory, practice, and prospects. Evolutionary Computation, 6(3):201-229, 1998.

[22] Robert Pool. Forget silicon, try DNA. New Scientist, 151(2038):26-31, July 13, 1996.

[23] D. L. Robertson and F. G. Joyce. Selection in vitro of an RNA enzyme that specifically cleaves single-stranded DNA. Nature, 344(6265):467-468, March 29, 1990.

[24] Gerhard Steger. Thermal denaturation of double-stranded nucleic acids: Prediction of temperatures critical for gradient gel electrophoresis and polymerase chain reaction. Nucleic Acids Research, 22(14):2760-2768, July 25, 1994. Online server is found at the URL: http://www.biophys.uni-duesseldorf.de/POLAND/poland.html.

[25] Willem P. C. Stemmer. DNA shuffling by random fragmentation and reassembly: In vitro recombination for molecular evolution. Proceedings of the National Academy of Science, U.S.A., 91:389-391, 1994.

[26] Willem P. C. Stemmer. Rapid evolution of a protein by DNA shuffling. Nature, 370:389-391, 1994.

[27] Willem P. C. Stemmer. The evolution of molecular computation. Science, 270:1510-1510, December 1, 1995.

[28] Willem P. C. Stemmer. Sexual PCR and Assembly PCR. In Robert M. Meyers, editor, The Encyclopedia of Molecular Biology and Molecular Medicine, volume 5, pages 447-457. VCH, New York, 1996.

[29] Jack W. Szostak. In vitro genetics. Trends in Biochemical Sciences, 172(3):89-93, 1992.

[30] H. J. Thiesen and C. Bach. Target detection assay (TDA) - A versatile procedure to determine DNA-binding sites as demonstrated on SP1 protein. Nucleic Acids Research, $18(11): 3203-3209,1990$.

[31] C. Tuerk and L. Gold. Systematic evolution of ligands by exponential enrichment - RNA ligands to bacteriophage-T4 DNA-polymerase. Science, 249(4968):505-510, August 3, 1990.

[32] J. P. Vartanian, M. Henry, and S. WainHobson. Hypermutagenic PCR involving all four transitions and a sizeable proportion of transversions. Nucleic Acids Research, 24(14):26272631, July 15, 1996.

[33] Roy Williams. Data powers of ten. Web page at http://www.ccsf.caltech.edu/ roy/dataquan.

[34] David Harlan Wood, Junghuei Chen, Eugene Antipov, Walter Cedeño, and Bertrand Lemieux. A DNA implementation of the Max 1s problem. In Wolfgang Banzhaf, A. E. Eiben, Max H. Garzon, Vasant Honavar, Mark Jakiela, and Robert E. Smith, editors, GECCO-99: Proceedings of the Genetic and Evolutionary Computation Conference, July 13-17, 1999, Orlando, Florida USA., pages 1835-1842, San Francisco, 1999. Morgan Kaufman.

[35] M. Zaccolo, D. M. Williams, D. M. Brown, and E. Gherardi. An approach to random mutagenesis of DNA using mixtures of triphosphate derivatives of nucleoside analogues. Journal of Molecular Biology, 255(4):589-603, February 2, 1996. 
(D. Wood) Defartment of Computer Science, University of Delaware, Newark, DE E-mail address: нood@cis.udel.edu

URL: http://н日н.cis.udel.edu/ ${ }^{\sim}$ rood

(J. Chen) Dept. of Chemistry \& Biochemistry, University of Delaware, Newark, DE E-mail address: junghuei@udel.edu

URL: http://н日r.udel.edu/chem/chen/chen.html

(E. Antifov) Deft. of Chemical Engineering, University of Delaware, Newark, De E-mail address: ultra@udel.edu

URL: http://copland.udel.edu/ ${ }^{\sim} u l t r a /$

(B. Lemieux) Deft. of Plant \& Soll Sciences, University of Delaware, Newark, De E-mail address: blemieux@udel.edu

$U R L:$ http://bertrand ags .udel.edu

(W. Ceneño) Hewlett-Packard Company, 2850 Centerville Road, Wilmington, De E-mail address: rcedeno@lf.hp.com

URL: http://ннн.personal .psu .edu/users/н/x/нxc28 